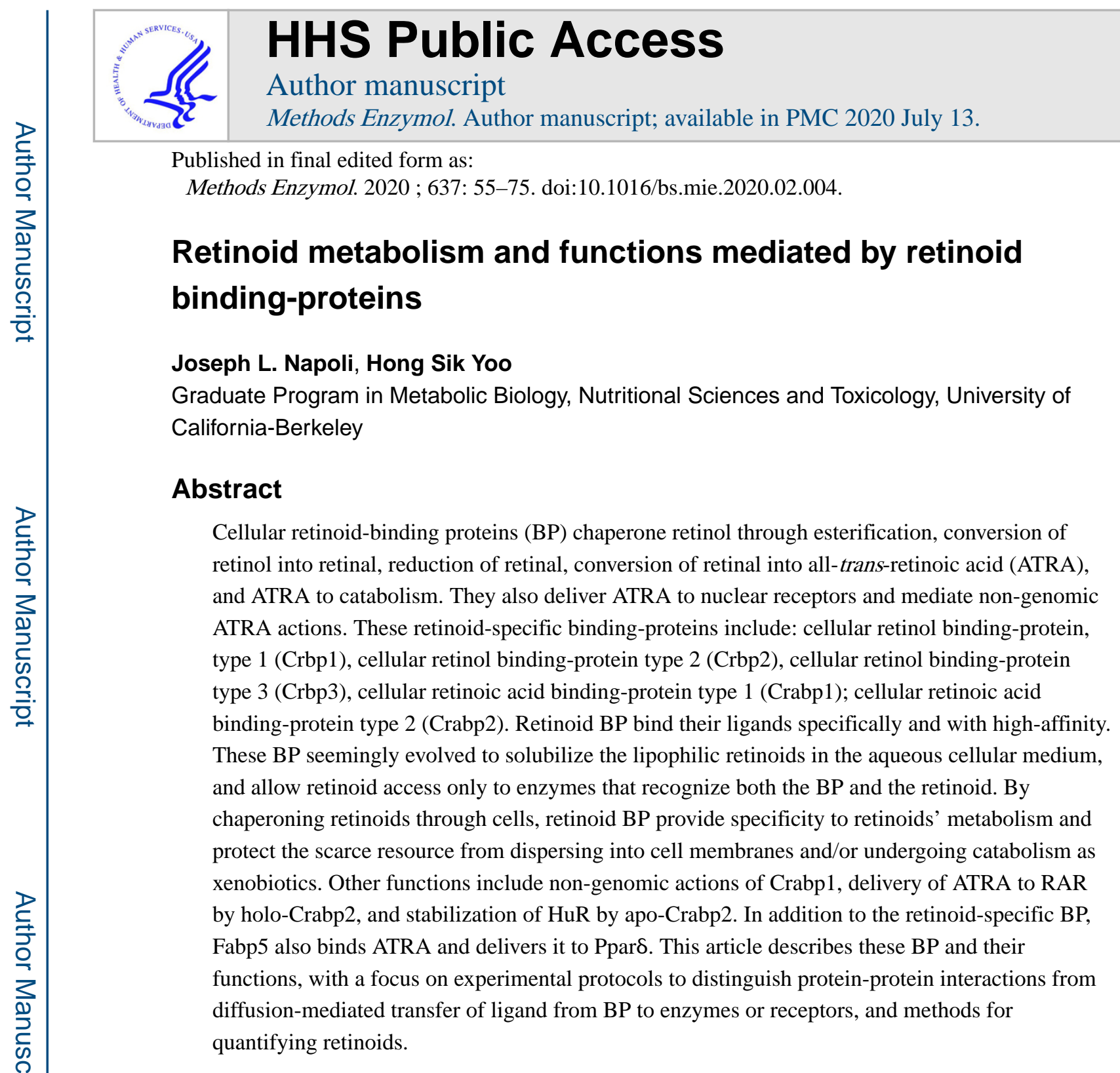

\title{
Keywords
}

retinol; retinoic acid; binding-proteins; retinol dehydrogenases; retinal dehydrogenases; retinal reductases; cytochrome P-450

\section{Nature of the BP}

Retinoid BP belong to the intracellular lipid binding-protein gene family and occur as flattened $\beta$-clam structures of $\sim 16 \mathrm{kDa}$. The Crbp structures enclose retinol and retinal deep within the protein with the functional groups (alcohols and aldehydes) projecting away from the entry portal (Figure 1). In Crabp, ATRA also orients with the functional group (a carboxyl) buried within the protein. In this case, however, the protein allows greater access to the $\beta$-ionone ring, where catabolism begins at the $\mathrm{C} 4$ and $\mathrm{C} 18$ carbons. Table 1 provides a brief summary of retinoid BP. 


\section{Crbp2 (encoded by rbp2)}

Crbp2, which accounts for $\sim 1 \%$ of the soluble protein in the small intestinal enterocyte, tightly controls retinoid absorption and metabolism (Ong, 1984; Ong et al., 1987, 1988;

Kakkad \& Ong, 1988). Diet provides pro-vitamin A carotenoids and retinyl esters, with the former prevailing quantitatively. A brush border lipase hydrolyzes luminal retinol esters to allow uptake of the released retinol, which binds with Crbp2 and undergoes lecithin:retinol acyltransferase (Lrat)-catalyzed re-esterification and incorporation into chylomicrons for systemic transport (Figure 2). Pro-vitamin A carotenoids undergo conversion into retinal catalyzed by the enzyme $\beta$-carotene oxidase type 1 (Bco1) (Harrison, 2012). The retinal formed binds to Crbp2. Crbp2 prevents dehydrogenation of retinal into ATRA, and directs retinal to reduction into retinol, which then undergoes esterification. Because carotenoids usually provide the major dietary source of vitamin $\mathrm{A}$, without direction into retinol rather than ATRA, they could cause ATRA toxicity upon metabolism. Thus, Crbp2 provides for efficient uptake of retinol by mass action and prevents retinoid toxicity by limiting ATRA biosynthesis.

The phenotype of $r b p 2$-null mice revealed another advantage of Crbp2. Rbp2-null mice pups die by $24 \mathrm{~h}$ after birth when delivered from dams fed a diet marginal in vitamin A (E et al., 2002). This indicates essentiality of Crbp2 in promoting efficient retinoid acquisition from diet and their systemic delivery.

\section{Crbp1 (encoded by rbp1)}

Crbp1 promotes retinol uptake, storage as retinyl esters and delivery of retinol for conversion into ATRA (Napoli, 2017). In liver, Crbp1 delivers retinol specifically to Lrat for esterification, and mediates transfer of retinol hepatocytes to stellate cells (MacDonald \& Ong, 1988). In extra-hepatic tissues, Crbp1 sequesters retinol transferred into cells by the membrane receptors for the serum Rbp (encoded by $r b p 4$ ), i.e. Stra6 and Rbpr2 (Berry et al., 2012) (Figure 2). By mass action and delivery of retinol to Lrat, Crbp1 ensures one-way uptake of retinol from serum. Crbp1 also delivers: retinol to retinol dehydrogenases (Rdh) for conversion into retinal; retinal to reductases (Rrd) for conversion into retinol; retinal to retinal dehydrogenases (Raldh) for conversion into ATRA.

Rbp1-null mice seem normal morphologically, but have low concentrations of retinyl esters in liver and eliminate them six-fold faster than wild-type mice (Matt et al., 2005). Disrupted hepatic storage likely relates to the function of Crbp1 in transferring retinol to stellate cells. Rbp1-null mice also have impaired glucose tolerance, as a result of pancreatic abnormalities (Kane et al., 2011). These include reduced islet expression of genes involved in glucose sensing and insulin secretion. The null mice also suffer from $\alpha$-cell infiltration into the $\beta$ cell field of pancreatic islets, which contributes to reduced glucose-stimulated insulin secretion, elevated glucagon secretion, increased gluconeogenesis and hyperglycemia. 


\section{BP contributions to retinoid metabolism}

\section{Crbp contributions to retinol and retinal metabolism}

Isolation of retinoids from the cellular milieu within Crbp1 (and Crbp2) with the hydroxyl function buried deeply in the BP and high affinity binding, begs the question of how enzymes can access these substrates. The issue was resolved by establishing that Lrat, retinol dehydrogenases ( $R \mathrm{dh}$ ), retinal reductases (Rrd), and retinal dehydrogenases (Raldh) interact with BP and "tease" retinoids from the BP into their active sites (Napoli, 2016). Several experiments have confirmed direct-transfer from BP to enzymes, i.e. no requirement for substrate diffusion through the aqueous medium. Michaelis-Menten kinetics ( $K_{m}$ and $V_{\max }$ values) of retinol and Crbp1-retinol with Lrat are similar (Ong et al., 1988). Moreover, apo-Crbp inhibits Lrat, but stimulates retinyl ester hydrolysis (Boerman \& Napoli, 1991; Herr \& Ong, 1992) (Figure 3). Likewise, the same kinetics are observed with Crbp1-retinal and Raldh1 and 2, i.e. unchanging Michaelis-Menten kinetics with either free retinal or Crbp1-retinal as substrate provider (Penzes et al., 1997; X. Wang et al., 1996). Apo-Crbp1 inhibits Raldh1, but not Raldh2. This suggests different contributions of Raldh1 and 2 to ATRA biosynthesis. Crbp1 also chaperones retinol to Rdh, whereas Crbp2 prevents Rdh access. In the case of retinal biosynthesis, $V_{\max }$ values of holo-Crbp1-delivered retinol are much lower than that of free retinol, perhaps indicating that the rate-limiting step involves retinol transfer from the BP to Rdh, i.e. that transfer occurs more slowly than the catalytic cycle (Posch et al., 1991). These data provide a teleological argument for direct BP participation in retinoid metabolism, insomuch as they indicate specific interactions with holo- vs apo-Crbp, and Crbp1 vs. Crbp2.

\section{Crabp contributions to ATRA catabolism}

Crabp1 and Crabp2 doubly-null-mice have a nearly four-fold higher death rate compared to wild type mice by six weeks after birth from unknown causes (Lampron et al., 1995). Nevertheless, the survivors seem normal morphologically, when fed a diet containing copious vitamin A, as occurs in rodent chow. The single observed exception involves a small outgrowth anomaly, predominantly in forelimbs, for both Crabp2 and the double knockout. Double null-mice do not have increased sensitivity to exogenous ATRA, suggesting that the $\mathrm{BP}$ are not required to protect against ATRA toxicity. Thus, ablations of Crabp1 and 2 have not clarified their functions.

The first and rate-limiting reactions of ATRA catabolism occur at $\mathrm{C} 4$ and $\mathrm{C} 18$, mediated by cytochrome P450 (Сyp)-catalyzed hydroxylation. Microsomal Cyp26a1, b1 and c1 function as the most dominant hydroxylases (Ross \& Zolfaghari, 2011; Topletz et al., 2012, 2015). ATRA induces these enzymes with exquisite sensitivity. Holo-Crabp1 increases catalytic efficiency 7 -fold $\left(k_{\mathrm{cat}} / K_{\mathrm{m}}\right)$ by delivering ATRA to catabolism catalyzed by microsomes, driven largely by a lower $K_{\mathrm{m}}$ value ( $<2 \mathrm{nM}$ for holo-Crabp1 vs. $\sim 50 \mathrm{nM}$ for free ATRA) (Fiorella \& Napoli, 1991). Both holo-Crabp1 and Crabp2 deliver ATRA to recombinant Cyp26b1 (Nelson et al., 2016). Cyp26a1 and c1 have not been tested. $K_{\mathrm{m}}$ values were lower with holo-Crabp1 $\left(K_{\mathrm{m}} \sim 22 \mathrm{nM}\right)$ and holo-Crabp2 $\left(K_{\mathrm{m}} \sim 24 \mathrm{nM}\right)$ delivery of ATRA to Cyp26b1, than with delivery of free ATRA $\left(K_{\mathrm{m}} \sim 65 \mathrm{nM}\right)$. Holo-Crabp delivery to Cyp26b1 enhances catalytic efficiencies $\left(k_{\text {cat }} / K_{\mathrm{m}}\right)$ of hydroxylation, generating relative values of 8 
and 12 for holo-Crabp1 and 2, respectively, vs 7 for unbound ATRA. With recombinant Cyp2b1, apo-Crabp1 and 2 added in graded amounts in excess of ATRA substantially reduce rates of catabolism. An equimolar amount of apo-Crabp1 relative to total ATRA reduces the $V_{\mathrm{m}}$ by $50 \%$, whereas apo-Crabp2 has less of an impact, reducing the $V_{\mathrm{m}}$ only by $\sim 25 \%$. Note, however, that even a 10-fold excess of apo-Crabp1 does not totally disable ATRA catabolism. Even a 3-fold excess of total Crabp to total ATRA, given the $k_{\mathrm{d}}$ values, would allow little to no free ATRA in the reaction mixture. Thus, Cyp26b1 must access ATRA bound with Crabp. Inhibition of catabolism by apo-Crabp may allow preservation of limited ATRA, consistent with holo-Crabp2 delivery of ATRA to nuclear RAR, and non-genomic regulatory functions of both Crabp1 and Crabp2.

\section{Fabp5 and ATRA function}

Fabp5 has lower affinity for ATRA $\left(k_{\mathrm{d}} \sim 42 \mathrm{nM}\right)$ than that of Crabp. Yet Fabp5 mediates

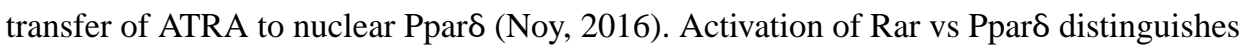
ATRA actions of inducing differentiation (Rar) from stimulating proliferation (Ppar反).

\section{Experimental Methods}

\section{Definition}

These protocols will address large-scale preparation of BP, evaluation of their integrity and the integrity of retinoids, application of kinetics to distinguish BP-chaperoned delivery through direct transfer of retinoids to acceptor proteins (enzymes and receptors) vs. diffusion-controlled transfer, and how to quantify retinoids in tissues and as products of metabolic reactions.

\section{Rationale}

These protocols relate up-to-date methods for handling and analytically rigorous quantification of retinoids with specificity and retinoid BP (Kane and Napoli, 2010). They include use of internal standards (so-called external standards should be avoided), verification of peak identity using HPLC/UV (internal standard needed) or mass fragmentation, reasons for obtaining total scans during UV assessment, and issues for developing sound kinetic data. Application of these techniques avoid spurious results.

HPLC with UV detection has relatively low equipment costs, relative to LC/MS/MS, can be specific for retinoids due to their unique absorbance spectra, but has a lower limit of quantification (LOQ) 0.4-1 pmol. HPLC/UV can work well with analyzing enzymatic production of ATRA and tissue concentrations of retinol and retinyl esters. GC/MS assays, with a lower limit of detection $<250 \mathrm{fmol}$, are more sensitive than HPLC/UV assays, but require ATRA derivatization. Methyl retinoate eluates only from packed GC columns, not from capillary GC columns. LC/MS/MS assays based on triple-quadrupole instruments offers sensitivity, specificity, no requirement for derivatization, and mass identification. The fragments analyzed and internal standards used must be chosen to avoid interfering peaks.

Substitutes for the analytically validated assays described here, such as "reporter" assays lack specificity and quantification. Expression assays that rely on Rar-driven reporters are 
not analytically rigorous, are not specific for ATRA, are not especially sensitive, and can produce both false-positive and false-negative results. These assays do not detect retinoids in real time because they reflect the time it takes to activate the receptor and generate the reporter, which can occur well after the retinoid has been catabolized.

\section{Materials, equipment and reagents}

Specialized equipment includes HPLC/UV, LC/MS/MS, shaking incubator (for E. coli production of BP), UV spectrophotometer, temperature controlled water bath (for kinetic studies). Specialized regents include vectors to generate recombinant BP and enzymes, available from labs referenced herein. A variety of retinoid internal standards can be used, such as 4,4-dimethyl-ATRA, 3,3-dimethyl-ATRA, and 3,4-didehydroretinal (for retinal quantification). Pentadeuterated ATRA should be used with caution. It served as a valid internal standard with GC/MS, but depending on the mass transitions used it can co-migrate with inferring peaks on LC/MS, rendering it invalid as an internal standard. LC/MS grade solvents should be used routinely. Only glass or stainless steel containers, pipettes, and syringes should be used to handle retinoids, as retinoids adhere to other surfaces, even Teflon. Gasses to evaporate samples should be applied via rubber tubing (not Tygon) to avoid contaminating samples with plasticizers. This is especially important for MS analyses.

\section{Protocols}

\section{BP preparation}

Recombinant production provides the most efficient method for pure obtaining apo- and holocellular retinoid BP in larger amounts (Levin et al., 1988; Napoli et al., 1998). This approach allows generation of ten's of $\mathrm{mg}$ of nearly pure protein. The BP should be stored at $-80{ }^{\circ} \mathrm{C}$ as apo-proteins. On the day of use, an aliquot can be divided into two parts. One should remain as apo and the other can be charged with excess ligand to ensure complete saturation, and then "dropped" through a Sephadex G-25 column, with a buffer containing freshly added sulfhydryl group protecting reagent, such as dithiothreitol. The holo-BP elute rapidly, given their sizes, free of unliganded retinoids, which remain on the column. This allows preparing precise mixtures of apo- and holo-BPs.

Retinol bound to holo-Crbp1, even stored at $-80{ }^{\circ} \mathrm{C}$, partially isomerizes to 9-cis-retinol over a month. When used for assaying ATRA biosynthesis, this gives the appearance of ATRA, because ATRA and 9-cis-RA closely migrate on LC columns and/or gives the false impression that holo-Crbp1 somehow generates 9-cis-RA. The ratios of the elution time of an internal standard with the elution times of ATRA vs 9-cis-RA remain constant, and can be used to distinguish one retinoid from the other, even if only one peak is present.

With BP isolated from tissues, exact apo- to holo- ratios cannot be determined without saturating an aliquot with the cognitive ligand and comparing UV spectra before and after saturation. Some reports have attempted ligand removal by irradiating the BP to destroy retinoids, or by stirring a layer of ether (in the cold) over the BP in aqueous buffer to extract retinoids. In our hands, these approaches destroyed/removed the retinoid, but also denatured Crbp, as indicated by attempting to re-charge an aliquot to determine whether the BP 
retained capacity to bind retinol. It did not. Without this verification by UV analyses, it is easy to mislead oneself into believing that the absence of retinoid leaves behind an intact apo-BP.

\section{Retinoid purification}

Retinoids should be handled under red or yellow light. Fluorescent white light and sunlight can isomerize and degrade retinoids within minutes. Retinol should be purified by HPLC before use. We find that impurities in retinol inhibit ATRA biosynthesis, especially in cell lines. On the other hand, retinol can be contaminated with retinal, which generates ATRA and gives the impression of Rdh activity.

\section{UV analyses}

UV analyses provides the "gold-standard" of retinoid and BP purities and quantitation. Weighing is inaccurate, given the quantities used and impurities in most commercial retinoids that do not absorb in the UV range.

Retinol has an $A_{\max }$ at $325 \mathrm{~nm}$ in ethanol with an $\varepsilon$ (molar absorptivity) value of 52,770 (Figure 4). ATRA has an $A_{\max }$ at $350 \mathrm{~nm}$ and an $\varepsilon$ value of 45,300. Retinal has an $A_{\max }$ at $383 \mathrm{~nm}$ and an $\varepsilon$ value of 42,880 . The $\mathrm{A}_{\max }$ values of trans retinoids distinguish them from geometric isomers, which have lower $A_{\max }$ values (National Research Council, 1972). A PDF with UV spectra information can be downloaded from https://www.nap.edu/catalog/ 21491/specifications-and-criteria-for-biochemical-compounds-third-edition.

The UV spectra of holo-Crbp1 and Crabp1 indicate quality and quantity by the $\mathrm{A}_{\max }$ and the ratio $A_{\max } / A_{\min }$, which should be 1.7 for a pure protein (Ong \& Chytil, 1978a, 1978b). Complete scans from at least $220 \mathrm{~nm}$ to $420 \mathrm{~nm}$ should be recorded, rather than just assessing absorbance at the peak. The quality of the complete spectrum reflects the quality of the sample. Spectra with "filled in" absorbance between 220 and $300 \mathrm{~nm}$ can indicate an impure sample, or an impure solvent.

\section{Tissue, cell and homogenate extraction}

Retinoid tissue and serum concentrations should be compared intra-strain, among animals fed the same diets, and in either the fasted or fed states (Obrochta \& Napoli, 2014; Obrochta, Krois, Campos \& Napoli, 2015). The nature of the diet (chow vs. AIN formula), the length of time fed the diet (first generation or subsequent generations), the mouse strain, and the timing of sample collection all affect concentrations of tissue retinoids, especially ATRA.

The extraction procedure has been modified from that originally published to provide a "cleaner" extract (Kane, Folias, Wang, et al., 2008a).

1. Add $1.5 \mathrm{~mL}$ methanol in a ground glass hand homogenizer (Duall, size 22 or similar, Kontes).

2. Add $\sim 100 \mathrm{mg}$ tissue and homogenize using a ground glass pestle with a motorized homogenizer (Heidolph). 
3. Transfer the homogenate into a glass conical centrifuge tube and add $10 \mu \mathrm{L} 1 \mu \mathrm{M}$ 3,3-dimethyl-ATRA (or other internal standard) and $10 \mu \mathrm{L} 50 \mu \mathrm{M} 3,4-$ didehydroretinol (internal standard for retinol). Vortex $10 \mathrm{sec}$.

4. Centrifuge $5 \mathrm{~min}$ at $1,200 \times \mathrm{g}$. Transfer the supernatant to a round-bottom glass tube.

5. To recover retinol and retinyl esters, add $100 \mu \mathrm{L} 0.5 \mathrm{M} \mathrm{KOH}$ in methanol. Vortex $10 \mathrm{sec}$.

6. Add $10 \mathrm{~mL}$ hexane. Vortex $20 \mathrm{sec}$. Centrifuge $3 \mathrm{~min}$ at $1,200 \times \mathrm{g}$.

7. Transfer the upper hexane phase to a clean glass tube, and evaporate the solvent under a stream of $\mathrm{N}_{2}$ or Ar.

8. Add $40 \mu \mathrm{L}$ methanol. Vortex $20 \mathrm{sec}$. Centrifuge.

9. Transfer the supernatant to an HPLC vial insert. Inject $20 \mu \mathrm{L}$ onto the column. HPLC/UV quantifies retinol and retinyl esters as published (Kane, Folias, \& Napoli, 2008).

10. To recover RA, add $150 \mu \mathrm{L} 4 \mathrm{M} \mathrm{HCl}$ to the remaining bottom phase. Vortex 10 sec.

11. Add $10 \mathrm{~mL}$ hexane. Vortex $20 \mathrm{sec}$. Centrifuge $3 \mathrm{~min}$ at $1,200 \times \mathrm{g}$.

12. Transfer the upper hexane phase to a clean round-bottom glass tube and evaporate the hexane under a stream of $\mathrm{N}_{2}$ or Ar.

13. For HPLC/UV add $40 \mu \mathrm{L}$ methanol to the tube. Vortex $20 \mathrm{sec}$. Centrifuge $3 \mathrm{~min}$ at $1,200 \times \mathrm{g}$. Transfer the supernatant to a HPLC vial insert. Inject $\sim 10 \mu \mathrm{L}$ onto the LC column.

14. For GC/MS or LC/MS/MS transfer the hexane (upper) phase to a glass tube and evaporate the solvent with a stream of $\mathrm{N}_{2}$ or Ar with heating at 25 to $30^{\circ} \mathrm{C}$ in a water bath. Suspend the residue in $40 \mu \mathrm{l}$ acetonitrile. Inject $5 \mu \mathrm{l}$ onto the LCMS/MS column.

Typically 15 to $35 \mathrm{mg}$ tissue or 100 to $200 \mu \mathrm{l}$ serum from adult mice generate reliable data (Napoli et al., 1985; Kane et al., 2005; Kane, Folias, Wang, et al., 2008a).

\section{Chromatography}

Multiple HPLC systems can resolve retinol and its esters (Kane, Folias, \& Napoli, 2008). The choice depends on the goal—quantifying retinol vs distinguishing retinol and ATRA isomers. For example a normal-phase HPLC system with UV detection resolves most common retinoids (Figure 5A). An ultra-high performance LC reverse-phase system with MS/MS detection can quantify retinal. This system has a 20 fmol lower limit of quantification (Figure 5B) (J. Wang et al., 2015). RA isomers can also be quantified with a LC reverse-phase system with MS/MS detection (Figure 5C). For the system shown, a 0.75 fmol lower limit of quantification can be achieved when assaying tissues (Kane, Folias, Wang, et al., 2008b). 


\section{Kinetic studies}

Complete Michaelis-Menten curves should be generated to truly assess enzymes' activity with substrates, especially when assessing whether a BP delivers retinoid through proteinprotein contact to an enzyme. This involves a range of concentrations that surround the inflection point; the $K_{\mathrm{m}}$ value for Michaelis-Menten kinetics. Failure to include points that extend beyond the inflection point generates unreliable kinetic constants. Data should be generated in a linear range of time and amount of protein. Because enzyme-catalyzed reactions produce product proportionally to substrate concentration, large decreases in the substrate concentration during assay also will produce unreliable kinetic constants. Therefore, Michaelis-Menten kinetics rely on the steady-state assumption, which postulates little change in substrate concentration during the assay. No change is impractical, so a "rule of thumb" relies on less than a $10 \%$ decrease in substrate. This produces data well within the margin of analytical variation. The rate of an especially active enzyme can be reduced by decreasing the assay temperature from $37{ }^{\circ} \mathrm{C}$ to $25{ }^{\circ} \mathrm{C}$, which decreases the rate $\sim 8$-fold, as was done for Raldh1 (Penzes et al., 1997). To skip these steps risks generating faulty kinetic values or even completely misleading data. A case in point involved Dhrs4 (aka Rrd) (Lei et al., 2003). This enzyme appeared to function as a dehydrogenase when assayed at one protein concentration and one time. Time-course and protein concentration experiments, however, showed no increase in retinal production from retinol as time and protein increased, indicating non-enzymatic (i.e. artifactual) retinal production. As much retinal was recovered at time 0 as after a $15 \mathrm{~min}$ assay. This illustrates the propensity of retinol to oxidize chemically. When assayed as a retinal reductase, Dhrs4 behaved as a typical enzyme, with increasing product formation as protein concentrations and assay times increased.

Several approaches can reveal whether an enzyme recognizes only free retinoid or also interacts with a BP to tease the retinoid into its active site. The easiest protocol simply uses a slight excess of BP to total retinoid as substrate, to ensure total retinoid (substrate) sequestration by the BP. If a substantial reaction occurs, the enzyme must recognize the "cassette" of holo-BP and remove the retinoid into its active site via protein-protein interaction. This ensues because the off-rate of retinoids from BP occur exceedingly slowly without an "active" receptor, and the affinity of retinoids with BP results in nearly total sequestration. With total BP $\sim 1$ to total retinoid $\sim 0.9$, virtually no free retinoid occurs in the assay mixture. Significant product formation must result from the enzyme recognizing the BP. Examples of this include interactions of Crbp1-retinol with Lrat and Crbp1-retinal with Raldh2 (Figure 6A).

A complication arises when using apo-Crbp1 in excess of retinoid, if the apo-BP inhibits the enzyme. The impact of apo-Crbp1 on Raldh1 provides an example of this (Figure 6B). In this case a lower $V_{m}$ in the presence of excess Crbp1 vs retinal (2:1) gave an impression of a lower $V_{m}$ with the BP. The Dixon plot (right) allowed extrapolation to a theoretical value of zero apo-Crbp1, and showed that the $V_{\mathrm{m}}$ with a precise 1:1 ratio of Crbp1 to retinal was nearly equivalent to that of the reaction with free retinal. These data allow two conclusions: 1) Raldh1 recognizes holo-Crbp1 and accesses its retinal; 2) apo-Crbp1 inhibits Raldh1, in contrast to Raldh2. 
A third kinetic display occurs if the rate-limiting event in the reaction becomes transfer of retinoid from the BP to the enzyme. The reaction with Rdh1 provides an example of this (Posch et al., 1992). In this case, presentation of holo-Crbp1 lowered the $V_{\mathrm{m}}$ of Rdh1 (not shown). Yet two different ratios of total Crbp1/retinol both generated Michaelis-Menten kinetics with nearly the same $K_{\mathrm{m}}$ and $\mathrm{V}_{\mathrm{m}}$ values (Figure 6C). Because the increase in Crbp1 would substantially lower the amount of free retinol present (if any), these data indicate that Rdh1 recognizes holo-Crbp1 and can tease retinol into its active site; otherwise there would have occurred a substantial decrease in $V_{\mathrm{m}}$ with increased apo-Crbp1. These data can be accompanied by a titration of apo-BP into the reaction mixture to determine whether inhibition also occurs. In the case of Rdh1, inhibition by apo-Crbp1 did not occur until higher concentrations of apo-Crbp1 were reached (not shown). Incidentally, using holoCrbp1 to deliver retinol allows no artifactual generation of retinal, whereas adding retinol to an aqueous homogenate generates retinal in the absence of enzyme.

apo-Crabp1 titration into assessment of ATRA catabolism, catalyzed by recombinant Cyp26B1, illustrates inhibition by BP, as the BP also serves as substrate (Nelson et al., 2016). Fifty nM ATRA provided the substrate in this reaction (Figure 6D). These data illustrate several points. At equimolar amounts of Crabp1/ATRA, the reaction rate decreased by $50 \%$. This alone does not necessarily indicate inhibition, but may indicate a shift in the rate-limiting event from catalysis of hydroxylation to ATRA release from holo-Crabp1. A further decrease in reaction rate with added apo-Crabp1 beyond a 1:1 ratio with ATRA indicates inhibition by apo-Crabp1. Note, however, that inhibition never reaches $100 \%$. Even at the ratio Crabp1/ATRA (10/1), 4-hydroxylation proceeds. Given the $k_{\mathrm{d}}$, virtually no free ATRA should occur even at a 1:1 ratio. Thus, these data show that Cyp26B1 can access ATRA bound to Crabp1 and that apo-Crabp1 inhibits Cyp26B1.

Another kinetic approach to distinguish diffusion-contingent transfer of ligand from BP to acceptor vs chaperoning by direct contact, relies on kinetics generated by changing the acceptor's concentration. The exchange of ATRA between Crabp1 and Rara vs Crabp2 and Rara illustrates this approach (Dong et al., 1999). Both Crabp1 and 2 have high affinity and lethargic off-rates, with Crabp1 having about twice the affinity of Crabp2. Crabp1 has a $k_{\text {off }}$ value of $0.22 \mathrm{~min}^{-1}$ and a $K_{\mathrm{d}}$ value of $0.06 \mathrm{nM}$, whereas the values for Crabp2 equal 0.42 $\min ^{-1}$ and $0.13 \mathrm{nM}$, respectively. Given the slow off-rates, little to no ATRA would occur in solution during the course of the experiment. If diffusion were the mechanism of transfer, increasing the acceptor concentration (Rara) should not alter the rate of transfer, as observed for Crabp1 (Figure 6E). In contrast, increasing the concentration of acceptor (Rara) increases the rate of transfer from Crabp2, indicating that transfer occurs via protein-protein interaction.

\section{Analysis and statistics}

For quantifying retinoids in model animal tissues, a minimum of 6 animals should be assayed; many more are desirable. Extensive biological variation occurs even with so-called genetically identical strains. Back-crossing is essential in using knockouts, as different strains of mice have different set-points of retinoid concentrations (Obrochta and Napoli, 2014). 


\section{Non-genomic BP functions}

HuR binds AU-rich sections in $3^{\prime}$-untranslated regions of mRNAs associated with cell proliferation, apoptosis and differentiation, and thereby protects transcripts from degradation, while regulating translation. Apo-Crabp2 binds to HuR, which enhances HuR affinity for mRNAs $>3$ orders of magnitude (Vreeland et al., 2014). ATRA binding changes the Crabp2 conformation, causing its release from HuR and exposing a nuclear localization signal (Sessler \& Noy, 2005). The holo-BP then undergoes SUMOylation, which prompts nuclear transfer and ATRA delivery to Rar (Majumdar et al., 2011). The newly formed apoBP rapidly returns to cytosol to re-bind HuR.

Rar do not medicate all ATRA functions. During embryonic stem cell stimulation by growth factors, such as EGF, competitive binding of holo-Crabp1 with cRAF dampens cell proliferation through decreasing ERK1/2 activation (Nagpal \& Wei, 2019). In contrast, in the absence of growth factor stimulation, holo-Crabp1 activates ERK1/2, which reduces cell growth by activating protein phosphatase $2 \mathrm{~A}$. Further, holo-Crabp1 induces differentiation by repressing Oct4, a gene essential for maintaining undifferentiated stem cells.

\section{Summary}

Despite discovery of the first retinoid BP more than 47 years ago, the field still has not developed comprehensive insight into their complex functions (Bashor et al., 1973). Clearly, retinoid BP contribute crucially to retinoid metabolism. Apparently, BP chaperone retinol, retinal and ATRA throughout cells, such that retinoids do not exist in their free forms. This has effects of solubilizing the lipophilic retinoids and securing specificity for their metabolism. Retinoid BP also contribute to non-genomic mechanisms of retinoid actions. BP ablations have not produced disastrous morphological consequences (perhaps with exception of Crbp1 on pancreas islets), but such observations do not support conclusions of physiological irrelevance. First, the BP undoubtedly promote efficient retinoid use. Vitamin A has been a scarce but essential nutrient and still remains the third most prevalent nutritional deficiency in many areas of the globe. Secondly, in general, BP knock-outs have not been subject to sufficient stress and observation to reveal other than gross morphological defects. Exceptions have included studies that have associated lack of Crbp1 with increased breast cancer and glucose metabolism abnormalities. Also, Crabp1-null mice have reduced basal cardiac function and cardiac hypertrophy, and suffer more damage than WT when challenged chronically with $\beta$-adrenergic agents (Park et al., 2018). The null mice also have arrested hippocampal neural stem cell proliferation, impacting memory formation. Moreover, reports continually increase of Crabp1 and 2 associations with incidences of various cancers and their prognoses.

\section{References}

Bashor MM, Toft DO, \& Chytil F (1973). In vitro binding of retinol to rat-tissue components. Proceedings of the National Academy of Sciences of the United States of America, 70(12), 34833487. [PubMed: 4519641] 
Berry DC, O'Byrne SM, Vreeland AC, Blaner WS, \& Noy N (2012). Cross talk between signaling and vitamin A transport by the retinol-binding protein receptor STRA6. Molecular and Cellular Biology, 32(15), 3164-3175. [PubMed: 22665496]

Boerman MH, \& Napoli JL (1991). Cholate-independent retinyl ester hydrolysis. Stimulation by Apocellular retinol-binding protein. The Journal of Biological Chemistry, 266(33), 22273-22278.

[PubMed: 1939249]

Cowan SW, Newcomer ME, \& Jones TA (1990). Crystallographic refinement of human serum retinol binding protein at 2A resolution. Proteins, 8(1), 44-61. [PubMed: 2217163]

Dong D, Ruuska SE, Levinthal DJ, \& Noy N (1999). Distinct roles for cellular retinoic acid-binding proteins I and II in regulating signaling by retinoic acid. The Journal of Biological Chemistry, 274(34), 23695-23698. [PubMed: 10446126]

E X, Zhang L, Lu J, Tso P, Blaner WS, Levin MS, \& Li E (2002). Increased neonatal mortality in mice lacking cellular retinol-binding protein II. The Journal of Biological Chemistry, 277(39), 3661736623. [PubMed: 12138113]

Fiorella PD, \& Napoli JL (1991). Expression of cellular retinoic acid binding protein (CRABP) in Escherichia coli. Characterization and evidence that holo-CRABP is a substrate in retinoic acid metabolism. The Journal of Biological Chemistry, 266(25), 16572-16579. [PubMed: 1653241]

Harrison EH (2012). Mechanisms involved in the intestinal absorption of dietary vitamin A and provitamin A carotenoids. Biochimica Et Biophysica Acta, 1821(1), 70-77. [PubMed: 21718801]

Herr FM, \& Ong DE (1992). Differential interaction of lecithin-retinol acyltransferase with cellular retinol binding proteins. Biochemistry, 31(29), 6748-6755. [PubMed: 1322170]

Kakkad BP, \& Ong DE (1988). Reduction of retinaldehyde bound to cellular retinol-binding protein (type II) by microsomes from rat small intestine. The Journal of Biological Chemistry, 263(26), 12916-12919. [PubMed: 3417642]

Kane MA, Chen N, Sparks S, \& Napoli JL (2005). Quantification of endogenous retinoic acid in limited biological samples by LC/MS/MS. The Biochemical Journal, 388(Pt 1), 363-369. [PubMed: 15628969]

Kane MA, Folias AE, \& Napoli JL (2008). HPLC/UV quantitation of retinal, retinol, and retinyl esters in serum and tissues. Analytical Biochemistry, 378(1), 71-79. [PubMed: 18410739]

Kane MA, Folias AE, Wang C, \& Napoli JL (2008). Quantitative profiling of endogenous retinoic acid in vivo and in vitro by tandem mass spectrometry. Analytical Chemistry, 80(5), 1702-1708. [PubMed: 18251521]

Kane MA, \& Napoli JL (2010). Quantification of endogenous retinoids. Methods Mol. Biol 652, 1-54. [PubMed: 20552420]

Kane MA, Folias AE, Pingitore A, Perri M, Krois CR, Ryu JY, Cione E, \& Napoli JL (2011). CrbpI modulates glucose homeostasis and pancreas 9-cis-retinoic acid concentrations. Molecular and Cellular Biology, 31(16), 3277-3285. [PubMed: 21670153]

Lampron C, Rochette-Egly C, Gorry P, Dollé P, Mark M, Lufkin T, LeMeur M, \& Chambon P (1995). Mice deficient in cellular retinoic acid binding protein II (CRABPII) or in both CRABPI and CRABPII are essentially normal. Development (Cambridge, England), 121(2), 539-548.

Lei Z, Chen W, Zhang M, \& Napoli JL (2003). Reduction of all-trans-retinal in the mouse liver peroxisome fraction by the short-chain dehydrogenase/reductase RRD: induction by the PPAR alpha ligand clofibrate. Biochemistry, 42(14), 4190-4196. [PubMed: 12680773]

Levin MS, Locke B, Yang NC, Li E, \& Gordon JI (1988). Comparison of the ligand binding properties of two homologous rat apocellular retinol-binding proteins expressed in Escherichia coli. The Journal of Biological Chemistry, 263(33), 17715-17723. [PubMed: 3053716]

MacDonald PN, \& Ong DE (1988). A lecithin:retinol acyltransferase activity in human and rat liver. Biochemical and Biophysical Research Communications, 156(1), 157-163. [PubMed: 3178828]

Majumdar A, Petrescu AD, Xiong Y, \& Noy N (2011). Nuclear translocation of cellular retinoic acidbinding protein II is regulated by retinoic acid-controlled SUMOylation. The Journal of Biological Chemistry, 286(49), 42749-42757. [PubMed: 21998312]

Matt N, Schmidt CK, Dupé V, Dennefeld C, Nau H, Chambon P, Mark M, \& Ghyselinck NB (2005). Contribution of cellular retinol-binding protein type 1 to retinol metabolism during mouse 
development. Developmental Dynamics: An Official Publication of the American Association of Anatomists, 233(1), 167-176. [PubMed: 15765518]

Menozzi I, Vallese F, Polverini E, Folli C, Berni R, \& Zanotti G (2017). Structural and molecular determinants affecting the interaction of retinol with human CRBP1. Journal of Structural Biology, 197(3), 330-339. [PubMed: 28057518]

Nagpal I, \& Wei L-N (2019). All-trans Retinoic Acid as a Versatile Cytosolic Signal Modulator Mediated by CRABP1. International Journal of Molecular Sciences, 20(15).

Napoli JL, Pramanik BC, Williams JB, Dawson MI, \& Hobbs PD (1985). Quantification of retinoic acid by gas-liquid chromatography-mass spectrometry: Total versus all-trans-retinoic acid in human plasma. Journal of Lipid Research, 26(3), 387-392. [PubMed: 3989394]

Napoli JL, Sperkova Z, Sperka M, \& Fiorella PD (1998). Expression and purification of CRABPs from E. coli. Methods in Molecular Biology (Clifton, N.J.), 89, 105-110.

Napoli Joseph L. (2016). Functions of Intracellular Retinoid Binding-Proteins. Sub-Cellular Biochemistry, 81, 21-76. [PubMed: 27830500]

Napoli Joseph L. (2017). Cellular retinoid binding-proteins, CRBP, CRABP, FABP5: Effects on retinoid metabolism, function and related diseases. Pharmacology \& Therapeutics, 173, 19-33. [PubMed: 28132904]

National Research Council. (1972). Specifications and Criteria for Biochemical Compounds: Third Edition The National Academies Press.

Nelson CH, Peng C-C, Lutz JD, Yeung CK, Zelter A, \& Isoherranen N (2016). Direct protein-protein interactions and substrate channeling between cellular retinoic acid binding proteins and CYP26B1. FEBS Letters, 590(16), 2527-2535. [PubMed: 27416800]

Noy N (2016). Non-classical transcriptional activity of retinoic acid. Subcellular Biochemistry 81, 179-199. [PubMed: 27830505]

Obrochta KM, \& Napoli JL (2014). Effects of diet and strain on mouse serum and tissue retinoid concentrations. PLoS One 9, e99435. [PubMed: 24911926]

Obrochta KM, Krois CR, Campos B, \& Napoli JL (2015). Insulin regulates retinol dehydrogenase expression and all-trans-retinoic acid biosynthesis through FoxO1. The Journal of Biological Chemistry, 290(11), 7259-7268. [PubMed: 25627686]

Ong DE (1984). A novel retinol-binding protein from rat. Purification and partial characterization. The Journal of Biological Chemistry, 259(3), 1476-1482. [PubMed: 6537950]

Ong DE, \& Chytil F (1978a). Cellular retinol-binding protein from rat liver. Purification and characterization. The Journal of Biological Chemistry, 253(3), 828-832. [PubMed: 563865]

Ong DE, \& Chytil F (1978b). Cellular retinoic acid-binding protein from rat testis. Purification and characterization. The Journal of Biological Chemistry, 253(13), 4551-4554. [PubMed: 566269]

Ong DE, Kakkad B, \& MacDonald PN (1987). Acyl-CoA-independent esterification of retinol bound to cellular retinol-binding protein (type II) by microsomes from rat small intestine. The Journal of Biological Chemistry, 262(6), 2729-2736. [PubMed: 3818619]

Ong DE, MacDonald PN, \& Gubitosi AM (1988). Esterification of retinol in rat liver. Possible participation by cellular retinol-binding protein and cellular retinol-binding protein II. The Journal of Biological Chemistry, 263(12), 5789-5796. [PubMed: 3281946]

Park SW, Persaud SD, Ogokeh S, Meyers TA, Townsend D, \& Wei L-N (2018). CRABP1 protects the heart from isoproterenol-induced acute and chronic remodeling. The Journal of Endocrinology, 236(3), 151-165. [PubMed: 29371236]

Penzes P, Wang X, \& Napoli JL (1997). Enzymatic characteristics of retinal dehydrogenase type I expressed in Escherichia coli. Biochimica et Biophysica Acta, 1342(2), 175-181. [PubMed: 9392526]

Posch KC, Boerman MH, Burns RD, \& Napoli JL (1991). Holocellular retinol binding protein as a substrate for microsomal retinal synthesis. Biochemistry, 30(25), 6224-6230. [PubMed: 2059629]

Posch KC, Burns RD, \& Napoli JL (1992). Biosynthesis of all-trans-retinoic acid from retinal. Recognition of retinal bound to cellular retinol binding protein (type I) as substrate by a purified cytosolic dehydrogenase. The Journal of Biological Chemistry, 267(27), 19676-19682. [PubMed: 1527087] 
Ross AC, \& Zolfaghari R (2011). Cytochrome P450s in the regulation of cellular retinoic acid metabolism. Annual Review of Nutrition, 31, 65-87.

Sessler RJ, \& Noy N (2005). A ligand-activated nuclear localization signal in cellular retinoic acid binding protein-II. Molecular Cell, 18(3), 343-353. [PubMed: 15866176]

Topletz AR, Thatcher JE, Zelter A, Lutz JD, Tay S, Nelson WL, \& Isoherranen N (2012). Comparison of the function and expression of CYP26A1 and CYP26B1, the two retinoic acid hydroxylases. Biochemical Pharmacology, 83(1), 149-163. [PubMed: 22020119]

Topletz AR, Tripathy S, Foti RS, Shimshoni JA, Nelson WL, \& Isoherranen N (2015). Induction of CYP26A1 by metabolites of retinoic acid: Evidence that CYP26A1 is an important enzyme in the elimination of active retinoids. Molecular Pharmacology, 87(3), 430-441. [PubMed: 25492813]

Vaezeslami S, Mathes E, Vasileiou C, Borhan B, \& Geiger JH (2006). The structure of Apo-wild-type cellular retinoic acid binding protein II at $1.4 \mathrm{~A}$ and its relationship to ligand binding and nuclear translocation. Journal of Molecular Biology, 363(3), 687-701. [PubMed: 16979656]

Vreeland AC, Yu S, Levi L, de Barros Rossetto D, \& Noy N (2014). Transcript stabilization by the RNA-binding protein HuR is regulated by cellular retinoic acid-binding protein 2. Molecular and Cellular Biology, 34(12), 2135-2146. [PubMed: 24687854]

Wang X, Penzes P, \& Napoli JL (1996). Cloning of a cDNA encoding an aldehyde dehydrogenase and its expression in Escherichia coli. Recognition of retinal as substrate. The Journal of Biological Chemistry, 271(27), 16288-16293. [PubMed: 8663198]

Wang J, Yoo HS, Obrochta KM, Huang P, \& Napoli JL (2015). Quantitation of retinaldehyde in small biological samples using ultrahigh-performance liquid chromatography tandem mass spectrometry. Analytical Biochemistry, 484, 162-168. [PubMed: 26045160] 


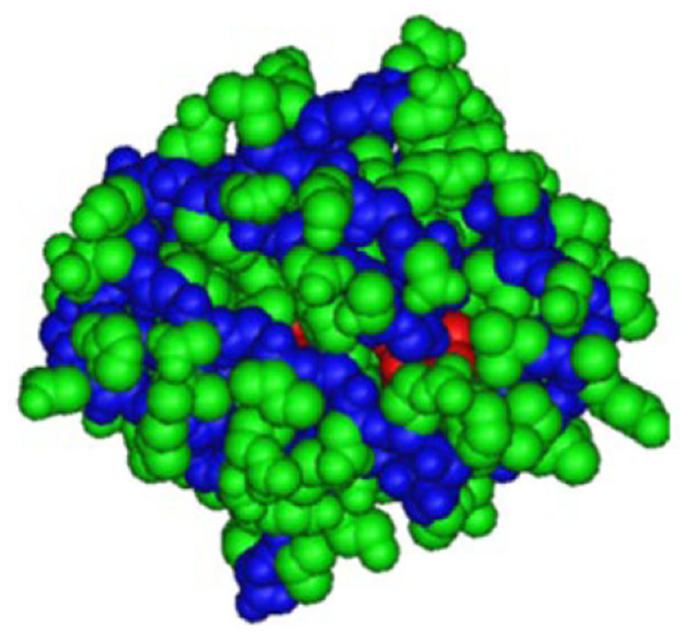

holo-Crbp1

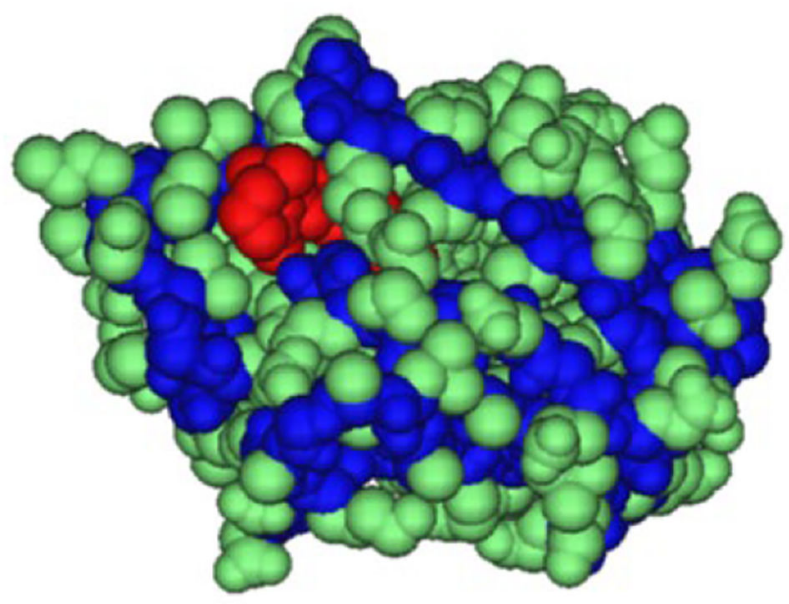

holo-Crabp2

Figure 1.

Space filling models of human cellular retinoid BP. The Crbp1 orientation shows the most visible aspect of retinol, with the red residues showing the $\beta$-ionone ring. The hydroxyl group buries itself inside the protein. The red residues in Crabp2 show the ATRA $\beta$-ionone ring. The carboxyl group buries itself inside the protein. In both cases, blue shows the protein backbones; green shows the protein side-chains. Structural information was downloaded from https://www.ncbi.nlm.nih.gov/Structure/CN3D/cn3dwin.shtml (Cowan et al., 1990; Vaezeslami et al., 2006; Menozzi et al., 2017). 

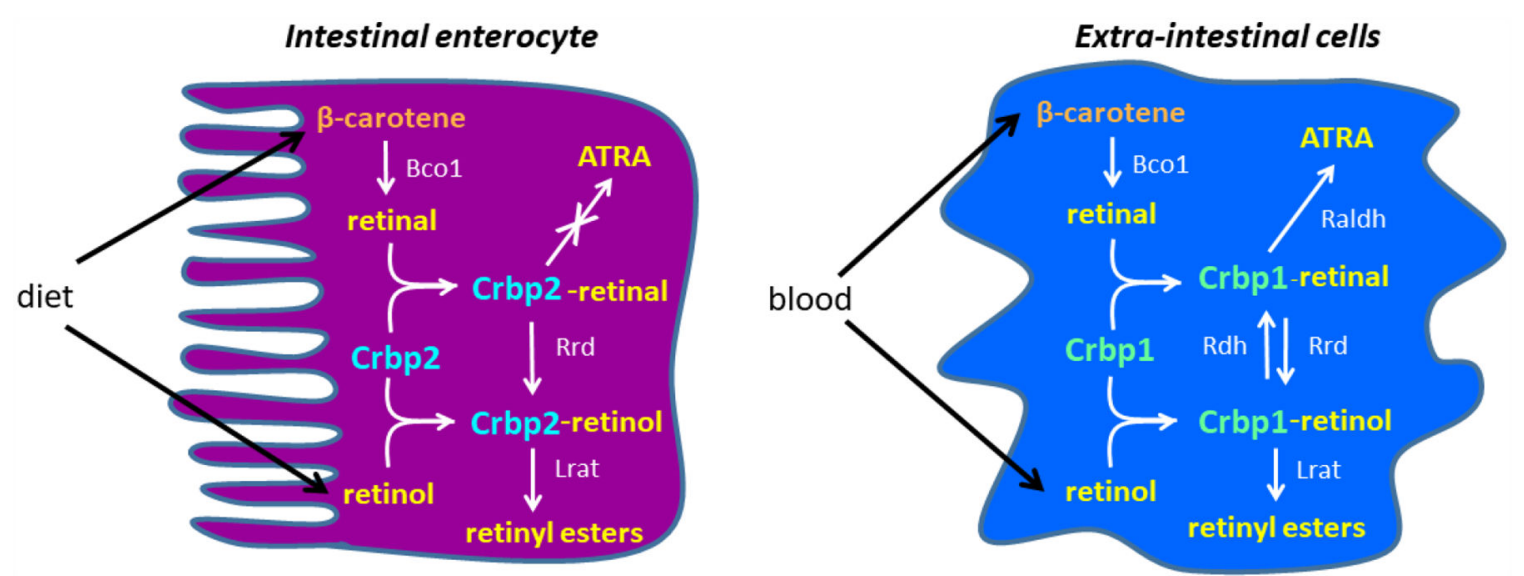

Figure 2.

Effects of Crbp1 and 2 on retinol and retinal metabolism. Crbp2 in the small intestine enterocyte prevents massive ATRA biosynthesis from retinal generated by dietary $\beta$-carotene cleavage. Outside the intestine, Crbp1 chaperones retinol and retinal for ATRA biosynthesis. Bco1, $\beta$-carotene oxidase type 1; Rrd, retinal reductase; Lrat, lecithin:retinol acyltransferase. 


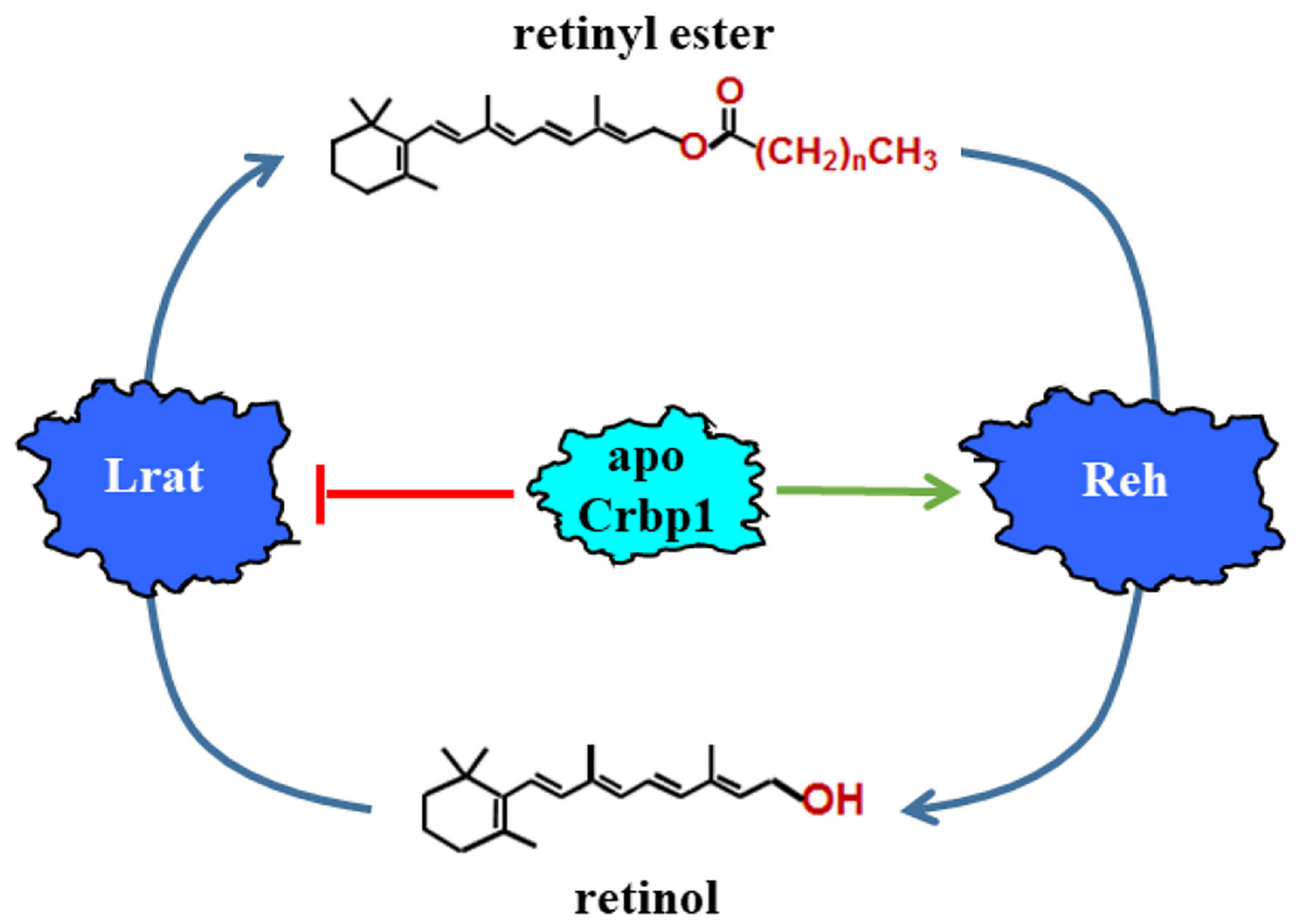

Figure 3.

Function of apo-Crbp1. The relative amounts of holo- and apo-Crbp1 reflect cellular retinol status, and thereby direct flux of retinol into and from retinyl esters. The mechanism involves apo-Crbp1 inhibiting Lrat and stimulating retinyl ester hydrolysis. In contrast, holoCrbp1 chaperones retinol into retinyl esters and into retinal for ATRA biosynthesis. 

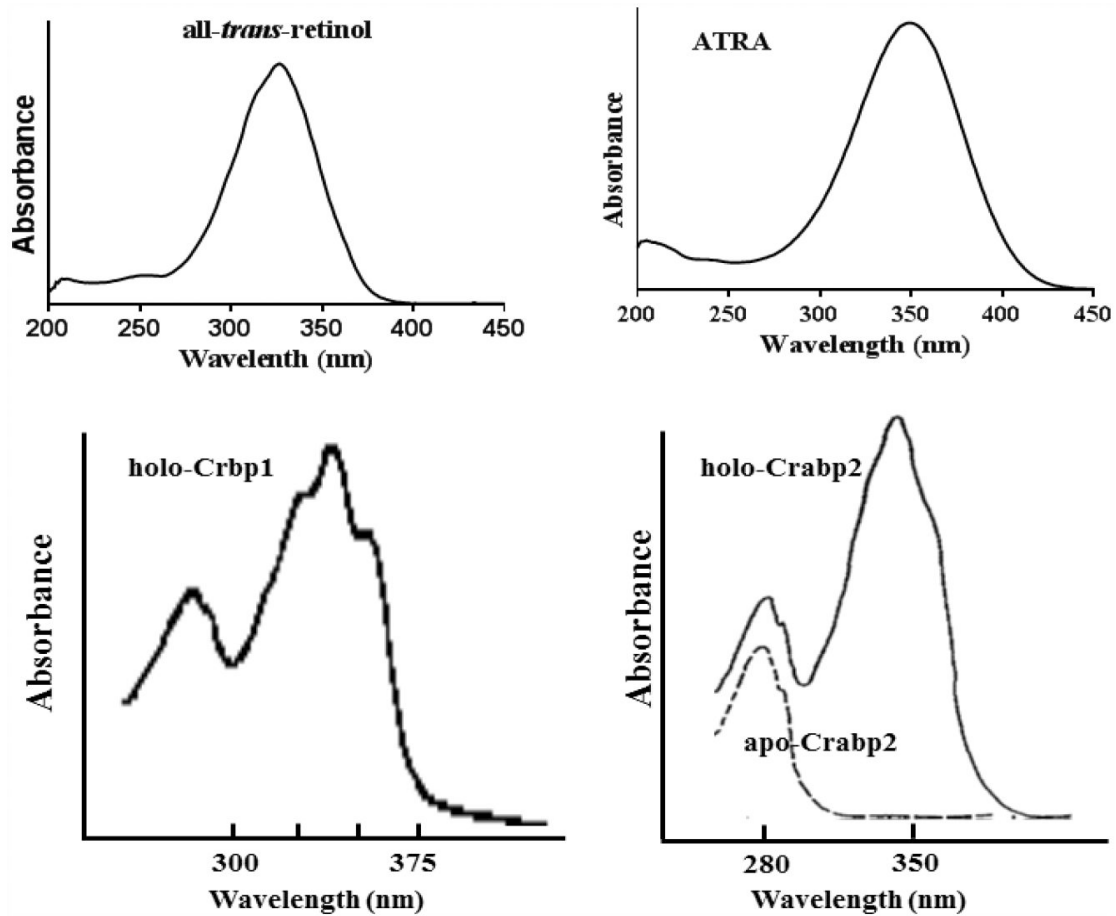

Figure 4.

UV spectra of retinoids and retinoid BPs. Spectra of retinoids were obtained in ethanol. The spectrum of holo-Crbp1 was taken in $20 \mathrm{nM}$ Hepes, $150 \mathrm{mM} \mathrm{KCl,} 2 \mathrm{mM}$ DTT at pH 7.5.

The $A_{\max }$ of holo-Crbp1 occurs at $350 \mathrm{nM}$. A ratio $\mathrm{A}_{250} / \mathrm{A}_{350}$ of 1.7 indicates a pure protein. The spectra of holo-Crabp1 (solid line) and apo-Crabp1 (dashed line) were obtained in 20 $\mathrm{mM}$ bis-Tris- $\mathrm{HCl}$ (pH 7.0), $2 \mathrm{mM} \beta$-mercaptoethanol, $0.05 \%$ sodium azide, $150 \mathrm{mM} \mathrm{NaCl}$ at pH 7.0. A molar absorptivity (ع) of 50,000 at $\mathrm{A}_{350}$ was used to quantify holo-Crabp1. 
A

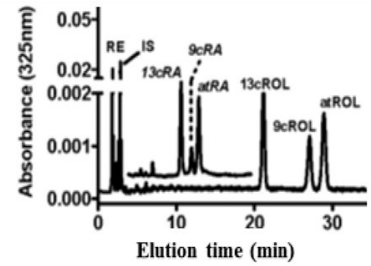

B

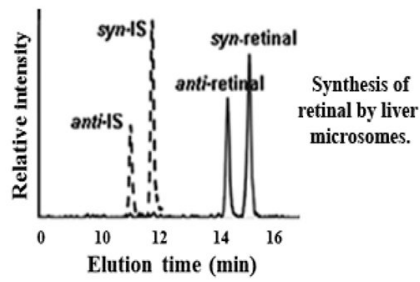

$\mathrm{C}$
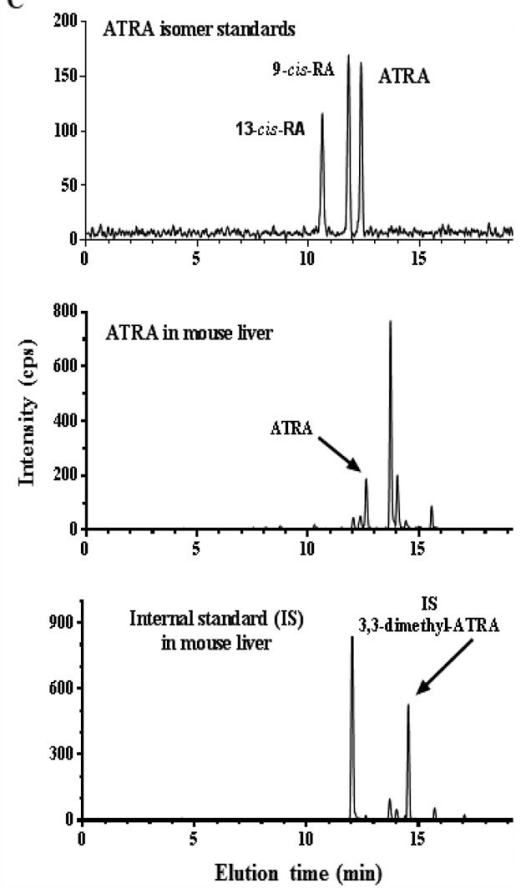

Figure 5.

Effects of BP on reactions/transfers. A) Crbp1 delivers substrates to Lrat and Raldh2 through channeling. The Lrat assay used a 1:1 ratio of BP/retinol. The Raldh2 assay used a ratio of 2:1 Crbp1/retinal. Both enzymes had similar $\mathrm{V}_{\mathrm{m}}$ values for bound and free retinoid, indicating that each accessed retinoid bound with BP. Lrat had similar $K_{\mathrm{m}}$ values for free (not shown) and bound retinol (MacDonald \& Ong, 1988). Crbp1 lowered the $K_{\mathrm{m}}$ value for retinal by $\sim 2$-fold, indicating a 2 -fold increased $k_{\text {cat }}$ value (X. Wang et al., 1996). B) Effects of Crbp1 on Raldh1 reaction kinetics. The assay used a ratio of 2:1 Crbp1/retinal. The intersect with the Y-axis in the Dixon plot (right) allowed extrapolation to a theoretical $\mathrm{V}_{\mathrm{m}}$ value with zero apo-Crbp1. C) Crbp1 delivers retinol to Rdh1. The lack of difference in the $\mathrm{V}_{\mathrm{m}}$ values with different amounts of total Crbp1 (increased apo-Crbp1) indicates Rdh1 can access retinol bound to Crbp1. D) Titration of apo-Crabp1 into ATRA catabolism catalyzed by recombinant Cyp26B1. Fifty nM ATRA were used (Nelson et al., 2016). 4-Hydroxy- 
ATRA was the metabolite monitored. Failure to completely prevent the reaction shows that Cyp26B1 recognizes BP and accesses its ATRA. E) Rates of ATRA transfer from Crabp to Rara. The increase in the rate as the Rara concentration increases indicates protein-protein interaction between the BP and the nuclear receptor (Dong et al., 1999). 
A
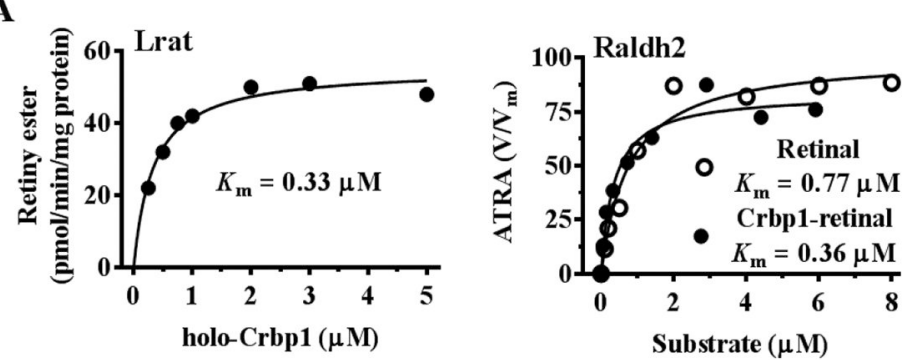

B
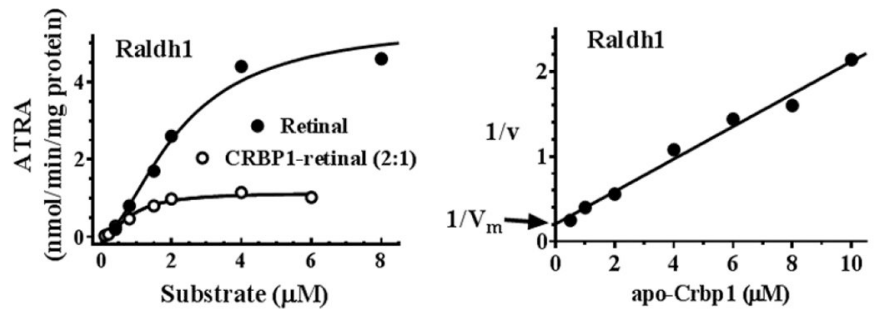

C
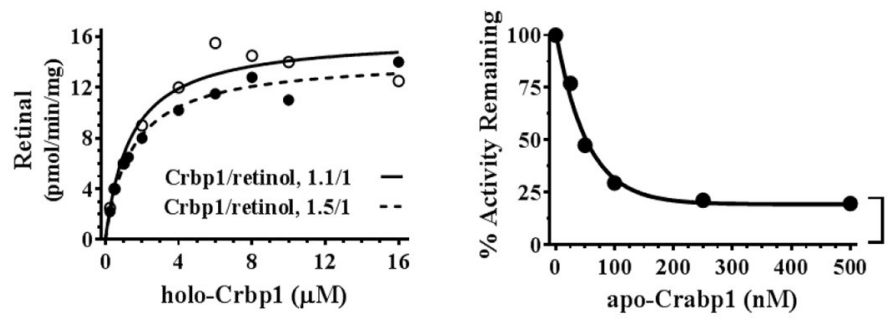

D
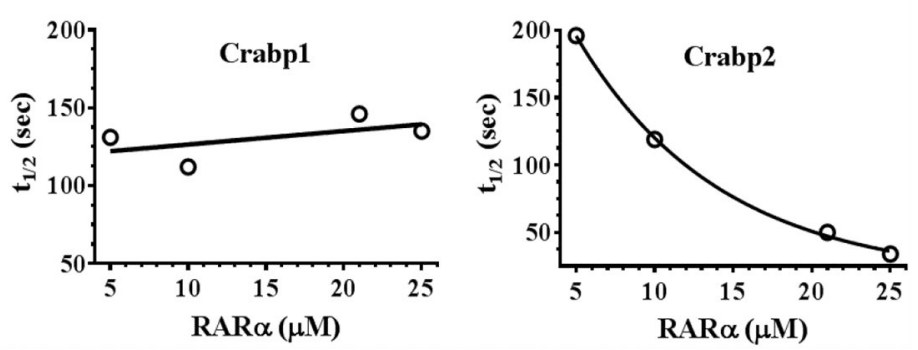

Figure 6.

Chromatographic resolution of retinoids. A) Normal phase HPLC separation of retinol and ATRA isomers with $0.4 \%$ 2-propanol/hexane with an elution of $2 \mathrm{ml} / \mathrm{min}$ : retinyl palmitate (RE) (2.0 min); internal standard (retinyl acetate), $3.6 \mathrm{~min}$; 13-cis-RA, $10.9 \mathrm{~min}$; 9-cis-RA, 12.1; ATRA, $13.1 \mathrm{~min}$; 13-cis-retinol, $20.9 \mathrm{~min}$; 9-cis-retinol, $27.0 \mathrm{~min}$; all-trans-retinol, $28.9 \mathrm{~min}$. Absorbance of retinol isomers was monitored at $325 \mathrm{~nm}$ (left y axis); absorbance in the overlay showing RA isomers was monitored at $340 \mathrm{~nm}$ (Kane, Folias, \& Napoli, 2008). B) Resolution of $s y n$ - and anti-all-trans-retinal oximes and oximes of the internal standard 3,4-didehydro-all-trans-retinal (IS). Ultra-high-performance LC used a C18 reverse-phase column $(2.1 \times 150 \mathrm{~mm})$ at a flow of $0.4 \mathrm{ml} / \mathrm{min}$. The column was eluted with $80 \% \mathrm{~A}$ (0 to $2 \mathrm{~min}$ ), 80 to $85 \%$ A ( 2 to $2.5 \mathrm{~min}$ ), 85 to $95 \% \mathrm{~A}$ (2.5 to $17.5 \mathrm{~min}$ ), 95 to $100 \%$ A (17.5 to $18 \mathrm{~min}$ ), $100 \%$ A (18 to $20 \mathrm{~min}$ ), 100 to $80 \% \mathrm{~A}$ (20 to $20.5 \mathrm{~min}$ ), re-equilibrating $3.5 \mathrm{~min}$. Mobile phase A: $0.1 \%$ formic acid in acetonitrile. Mobile phase B: $0.1 \%$ formic 
acid in water. Retinal oximes used a $\mathrm{m} / z 328.5$ to 236.4 transition; 3,4-didehydroretinal oximes used a $\mathrm{m} / \mathrm{z} 326.6$ to 234.4 transition (J. Wang et al., 2015). C) Resolution of ATRA from its isomers. An Ascentis RP-Amide column (Supelco, $2.1 \times 150 \mathrm{~mm}, 3 \mu \mathrm{m}$ ) was eluted at $0.4 \mathrm{ml} / \mathrm{min}$ with a linear gradient: $70 \% \mathrm{~A}(0-3 \mathrm{~min}) ; 70 \% \mathrm{~A}$ to $95 \% \mathrm{~A}(3-15 \mathrm{~min}) ; 95 \% \mathrm{~A}$ (15-20 $\mathrm{min}) ; 5 \%$ A to $70 \%$ A (20-21 min); re-equilibrate at 70\% A (21-25 min). RA isomers were monitored using a $\mathrm{m} / z 301.1[\mathrm{M}+\mathrm{H}]+$ to $\mathrm{m} / z 205.0$ transition; the IS, 3,3dimethyl-RA was monitored with a $\mathrm{m} / z 329.4[\mathrm{M}+\mathrm{H}]+$ to $\mathrm{m} / z 151.3$ transition. 
Table 1.

Cellular retinoid binding-proteins, distributions, ligands and their affinities

\begin{tabular}{|c|c|c|c|}
\hline Protein & Post-natal distribution & Ligands & $k_{\mathrm{d}}(\mathrm{nM})$ \\
\hline \multirow{4}{*}{ Crbp1 } & \multirow{4}{*}{$\begin{array}{c}\text { Ubiquitous? } \\
\text { (low in small intestine) }\end{array}$} & all-trans-retinol & $\unlhd$ \\
\hline & & 3,4-didehydroretinol & --- \\
\hline & & 9-cis-retinol & 11 \\
\hline & & all-trans-retinal & 9 \\
\hline \multirow{2}{*}{ Crbp2 } & \multirow{2}{*}{ High in small intestine } & all-trans-retinol & 10 \\
\hline & & all-trans-retinal & 11 \\
\hline \multirow{2}{*}{ Crbp3 } & \multirow{2}{*}{ Heart, muscle, adipose, mammary } & all-trans-retinol & 109 \\
\hline & & 9-cis-retinol & \\
\hline Crabp1 & Widespread & ATRA & 0.06 \\
\hline Crabp2 & Limited? (skin, uterus, and ovary) & ATRA & 0.13 \\
\hline \multirow{2}{*}{ Fabp5 } & \multirow{2}{*}{ Widespread } & ATRA & 35 \\
\hline & & Long-chain fatty acids & $15-20$ \\
\hline
\end{tabular}

